\title{
Editorial
}

\section{SAN AGUSTÍN: \\ MÁS DE CIEN AÑOS POR CELEBRAR Y MUCHO POR HACER}

\section{Viaje iniciático: entre el mito y la poesía}

Recuerdo mi primer viaje a San Agustín con el profesor Luis Ernesto Lasso, en el curso de Literatura Hispanoamericana, año 1985. La emoción que sentí desde el momento en que empezamos a ascender por entre las montañas majestuosas, entre cascadas y en el fondo el río Magdalena con sus aguas límpidas y rápidas. Sin saberlo, esta salida se convirtió para mí en un viaje de iniciación, en una experiencia vital que determinó mi relación con el pueblo, con el paisaje y con la gente que va mucho más allá de su valor arqueológico.

El profesor, apasionado defensor de la Cultura de Ullumbe, ${ }^{1}$ se empeñaba en que los estudiantes conociéramos y valoráramos nuestro patrimonio para que, como herederos de esta gran cultura, nos sintiéramos orgullosos y viéramos en ella una de nuestras señas de identidad, su consigna: «conozcamos nuestra herencia, salgamos de la opitud y de la minoría de edad, tallando nuestro sitio en el universo» (Lasso, 2005: 36).

Por aquella época el maestro Lasso contaba la historia de las estatuas que Preuss se había llevado y que permanecían olvidadas en los sótanos del Museo de Berlín, ante la indiferencia de todos; también de la lucha que dieron los habitantes del pueblo cuando un presidente pretendía llevarse las estatuas para «decorar» los jardines del Palacio de Nariño. El profesor luchó durante años por promover la Cultura Megalítica y dar elementos teóricos para su análisis y comprensión, es así como logró que instituyeran la Cátedra Agustiniana en el programa de Lengua Castellana y capacitó a maestros de Neiva, Garzón para que se convirtieran en impulsores y defensores de nuestro patrimonio. Su aporte ha sido grande y sus semillas seguirán germinando en lucha constante contra el olvido.

Después de este primer viaje, vinieron muchos más. En diferentes etapas de mi vida. En diferentes momentos del pueblo. A través de las continuas visitas he seguido sus transformaciones: lentas unas, vertiginosas otras. He observado un bosque de eucaliptos desaparecer para dar paso a casas de ladrillos; he advertido cómo los caminos y parajes por los que transitaban caballos cargados con mercados y campesinos, han sido invadidos por motos y carros que acallaron el silencio y el canto de las aves. San Agustín ha sobrevivido al olvido y al abandono aferrado a sus turistas, a sus artesanías, a la piedra que tallan como herederos de los antiguos artistas.

Viví con familias campesinas y disfruté de su compañía, de su sencillez, de su hospitalidad y de su humor taimado. Compartí sus sufrimientos, sus angustias, su pobreza y su lucha por subsistir. Para ellos el turismo es una palabra que no les dice mucho, una ilusión. Generaciones de niños y niñas han crecido y su proyecto de vida es convertirse en padres y madres que trabajan la tierra de otros como jornaleros, recolectores o mayordomos de las fincas de extranjeros y gamonales del pueblo.

1. En el transcurso del texto nos referiremos a la cultura agustiniana de diversas maneras: Cultura Ullumbe, Cultura Megalítica del Alto Magdalena 0 CulturaAgustiniana. 
En San Agustín no hay oportunidades. Hubo una vez un muchacho que logró entrar a la universidad, trabajaba duro durante las vacaciones en una fábrica donde se hacían réplicas de las estatuas y estudiaba con pasión para ser el mejor estudiante y conservar la beca. Se graduó con honores y regresó al para ser el rector de un colegio. Y otro más que quiere ser abogado para llegar a la alcaldía y hacer algo por su pueblo. Estas son excepciones. La mayoría de los jóvenes (hombre y mujeres), no tienen la posibilidad de ingresar a la universidad, aspirar a un trabajo bien remunerado, montar su propio negocio o cumplir con los requisitos para acceder a préstamos y hacer productivas las parcelas. Para ellos no existe Agro Ingreso Seguro.

Las viejas casas de bareque han sido demolidas para dar paso a las construcciones de ladrillo. El pueblo, se ha llenado de tiendas, de motos conducidas por dementes, de artesanos, de hermosos hoteles y buenas panaderías, excelentes restaurantes y bares que invitan a la buena música. De muchachos con pintas citadinas. En San Agustín el día lunes parece de fiesta es día de mercado. Los campesinos bajan a comprar las provisiones para la semana, las mujeres y los niños lucen su mejor ropa, los pequeños disfrutan de un helado y los hombres se emborrachan con cerveza o con guarapo. He visto en una banca del parque a un krisna y un franciscano conversar, mientras son observados por las esculturas silenciosas. La Semana Santa y los rituales de los yanaconas se mezclan de manera natural. Allí las razas se cruzan; el rock y las chirimías se funden; la bonanza y la escasez se observan.

Caminé por las montañas, a pie y a caballo, aspiré el aire frío y purificador; comí moras silvestres; recorrí los parques, en la mañana, al medio día, al atardecer; entré a su mundo poético y sostuve conversaciones con los chamanes jaguares. Con perseverancia y desnudando mi mente intenté descifrar su discurso lítico, su epopeya en piedra. Descubrí con asombro las serpientes deslizarse en el Lavapatas y la sangre de los niños correr entre animales sagrados. Ascendí al Alto del Lavapatas a interrogar al Doble yo o al yo desdoblado, en la cima de mis incertidumbres realicé rituales profanos cargados de angustia y desesperación buscando una conexión con el universo. La tierra palpita y su corazón nace dentro de mí.

La Chaquira, observatorio astral, los ojos solitarios de los chamanes permanecieron fijos por generaciones y aprendieron sus misterios y arcanos. El solsticio y el equinoccio, las lluvias y el sol, el verano y el inverno. Las siembras y las cosechas. Contemplo el cielo e interrogo a los astros. Me entregó a la contemplación. Nuevamente escucho el silencio y el canto del río, su queja, las montañas que a pesar de su monumentalidad imploran protección. ¿De quién?

Chaquira, centro de poder, santuario sagrado, ahí quiero volver siempre.

\section{Preuss, desde la conjetura europea}

Preuss perteneció a la escuela americanista de Berlín; viajó por primera vez a México en 1905 para realizar estudios de campo sobre las tribus ubicadas en la sierra de Nayarit, especialmente con los indios Coras y Huicholes, posteriormente regresa a Alemania en 1907, luego de 19 meses de trabajo intenso en tierras mexicanas. De nuevo en su oficina del museo organiza la información y en 1908 presenta los resultados de su investigación a través de diferentes artículos y ponencias en los Congresos Internacionales Americanistas. Para 1912 es nombrado director de las secciones norte y centroamericana del Museo Etnológico de Berlín.

En septiembre de 1913 Konrad Teodoro Preuss emprende el viaje desde Europa a Colombia. En Barranquilla inicia su periplo al corazón del Macizo Colombiano, navegando el Río Magdalena en barco a vapor de «rueda trasera» hasta Girardot; el recorrido se hizo en 12 días. Para esquivar los raudales de Honda, interrumpe el viaje por vía fluvial y desde la Dorada Caldas a Beltrán Cundinamarca se transporta en el ferrocarril. De Girardot a Purificación retoma el río en embarcaciones más pequeñas. Decide ir hasta Bogotá para comprar provisiones y conseguir un ayudante y guía, Telésforo González. De Purificación hasta San Agustín el viaje debe hacerse a lomo de mula, así que en Purificación 
organiza la expedición: compra las animales, contrata cargueros e inicia su travesía. El recorrido Purificación-San Agustín (pasando por Neiva donde permaneció un corto tiempo) transcurrió en 16 días. (Preuss, 1974: p.p. 35,46)².

Durante los cuatro meses que el profesor Preuss permaneció en San Agustín, exploró sus alrededores, clasificó, describió y catalogó 108 esculturas, excavó montículos y templetes, tomó fotografías, sacó moldes de varias esculturas. El 18 de febrero envía el primer cargamento de antigüedades indígenas hacia Neiva; en marzo despacha el segundo con varios moldes y 14 estatuas pequeñas. Las investigaciones de Preuss se realizan entre diciembre de 1913 hasta abril de 1914, dando inicio a la que se considera la primera expedición de carácter científico sobre la cultura megalítica del Alto Magdalena.

En 1919, finalizada la I Guerra Mundial, regresa a Alemania y cuatro años más tarde realiza una exposición para dar a conocer sus hallazgos: "Sólo en la primavera de 1923, después de que ya estaban terminados los vaciados en yeso de las grandes estatuas, pude reunir en una exposición todas estas extrañas obras de arte monumental en el gran patio del antiguo Museo de Artes y Oficios de Berlín». (Preuss. 1974:19). La exposición fue un éxito: traspasó las fronteras alemanas y llamó la atención de revistas y periódicos europeos. En 1929 aparece la obra Arte Monumental Prehistórico, Excavaciones en el Alto Magdalena y San Agustín, diez años después de que Konrad Preuss hubiera dejado Colombia. Estos dos sucesos marcan el inicio de la investigación arqueológica en San Agustín y en el país.

\section{Los predecesores de Preuss}

Es justo decir que antes de la llegada del etnólogo alemán a San Agustín, ya se tenía noticia de esta cultura cuyos registros se remontan al Siglo XVIII con Fray Juan de Santa Gertrudis en su obra Maravillas de la Naturaleza, quien hizo una lectura desde su visión religiosa e interpretó las figuras como «obispos de medio cuerpo hasta las rodillas, de piedra, con su mitra (...) de aquí fimos al otro monumento. Son cinco frailes franciscanos observantes...» para concluir que el origen de estas esculturas eran obra del demonio: «Yo sólo me persuado que el demonio los fabricaría, y me fundo en que en la India los indios no tenían fierro, y por consiguiente tampoco instrumentos para poderlos fabricar». (Llanos, 1995: 18).

En 1797 Francisco José de Caldas recorre la región y observa «con admiración el producto de las artes de esta nación sedentaria, de que nuestros historiadores no nos han transmitido la menor noticia»; así lo registra en el Semanario del Nuevo Reino de Granada. (Caldas, 1808: 35).

Luego, para el año 1857 el geógrafo Agustín Codazzi visita el Huila como parte de los estudios de la Comisión Coreográfica y llega hasta San Agustín. Descubre, describe y analiza 34 esculturas, las cuales son dibujadas por J.M. Paz; levanta un mapa del lugar y realiza un acercamiento que le permite hacer una interpretación de sus hallazgos. Sus análisis quedaron registrados en el texto Ruinas de San Agustín descritas i esplicadas por A. Codazzi, publicada por Felipe Pérez en Geografía física i política de los Estados Unidos de Colombia en 1863, como un Apéndice.

Para Codazzi la cultura que produjo las esculturas era «una nación bastante civilizada» y tenía «una teogonía completa y destinaban determinados lugares al culto de ídolos sedentarios». No duda del carácter religioso y sagrado del sitio y de lo que representaban sus monumentos: «es un lugar exclusivamente relijioso destinado a la celebración de ritos complicados que se relacionaban con la vida social i constituían una enseñanza dada por medio de la iniciación». (Codazzi, 1863).

Carlos Cuervo Marques, estuvo en San Agustín en 1892 y luego publica el libro Prehistoria y viajes americanos (1893) en el cual se registran sus observaciones sobre su visita; en 1920 publica en Madrid el libro Estudios

2. Sobre este recorrido circula por YouTube un video en alemán donde se presenta la expedición. http://www.youtube.com/watch?v=X_nfgowdt9U 
arqueológicos y etnológicos. Según sus estudios y observaciones, Cuervo Marques, considera que el pueblo escultor debió estar relacionado con otras regiones y otros centros de cultura.

Es importante hacer notar que Preuss no fue el «descubridor» de la cultura agustiniana; tampoco el iniciador de la investigación científica, porque ya Agustín Codazzi había realizado una sistematización e interpretación de las esculturas, la cual fue la base para la investigación del profesor alemán. Él mismo, en el capítulo I del libro Arte Monumental Prehistórico, pondera y valora el trabajo de Codazzi: «Por ello el primer descubridor de este adoratorio, el cartógrafo italiano Agustín Codazzi, tuvo la impresión, hace ya setenta años, de que se halla en un distrito sagrado, en donde los indios de remotísimas edades se iniciaban en los secretos arcanos de lo trascendental [...] podemos afirmar que, aunque hay algunas interpretaciones erróneas en la obra de Codazzi, él se nos presenta como un explorador concienzudo y serio. Todo nuevo investigador de San Agustín deber ir a buscar en él la base del trabajo que va a emprender». (Preuss, 1929: 31-32).

En consecuencia, en la celebración de los cien años de los descubrimientos científicos, no solo se debe reconocer las investigaciones hechas por Preuss, sino también los de sus antecesores, especialmente a Francisco José de Caldas, Agustín Codazzi y Carlos Cuervo Marques. Darle todos los créditos a Preuss, no es justo, teniendo en cuenta que su nombre no sale bien librado a través de los años, por el trasladado de las 14 piezas (según Barney Cabrera) 3 al Museo Etnológico de Berlín y que después de cien años todavía permanecen allí, ante la indiferencia del gobierno nacional, departamental y regional. Su imagen de investigador serio y respetable, se ha desdibujado en el imaginario del pueblo para convertirse en una especie de Indiana Jones, cazador de tesoros perdidos de los pueblos supuestamente primitivos, con cámara fotográfica, instrumentos de investigación, sombrero, gafas, pantalón y camisa caqui. Y una sed enorme de desentrañar la escritura lítica que desbordaba su imaginación.

\section{Y entonces, ¿cómo vamos a celebrar?}

Bienvenidas todas las actividades programadas para la celebración: conferencias, capacitaciones, conciertos, talleres, diálogos, las reimpresiones de los libros, las becas, los documentales, las inversiones; pero más allá de la coyuntura de la celebración convendría reflexionar sobre otros aspectos que aportarían de manera significativa al pueblo y a sus habitantes.

Es importante destacar la campaña que está llevando a cabo David Dellenback ${ }^{4}$ para repatriar las estatuas que dormitan en el Museo de Berlín y que por fin retornen a sus montañas sagradas. Para ello es necesario que los entes gubernamentales del Huila realicen las gestiones a nivel del gobierno nacional para que se inicien los procesos de la repatriación. Por otra parte, le corresponde al Icanh asumir ésta acción como una prioridad. Los académicos, periodistas, huilenses y agustinianos, estar atentos para que la iniciativa continúe y llegue a feliz término. Para ello usar las redes sociales, los periódicos, las aulas de clase y todos los espacios de socialización. Desarrollar talleres con la comunidad, con los niños y jóvenes y hacer visible esta situación.

Se han logrado grandes avances en la atención y en la prestación de servicios; hay guías profesionales que gracias a capacitaciones realizadas por el Sena, conocen a los estudiosos de San Agustín y enriquecen sus recorridos con las historias orales heredadas de sus abuelos o del saber recogido en el acompañamiento que han hecho algunos investigadores. Las cabalgatas son organizadas y dirigidas por experimentados rumberos, quienes

3. No se conoce a ciencia cierta cuántos fueron las esculturas que Preuss se llevó; Barney Cabrera en las notas y observaciones del libro Arte Monumental registra como 14 figuras líticas, David Dellenback asegura que son 21 de San Agustín y 14 de Nariño; Juan Friede sostiene que son 27.

4. David Dellenback es uno de los extranjeros que ha hecho suya la cultura agustiniana, lleva más de treinta años viviendo en San Agustín. Este año publicó el libro Las estatuas del pueblo escritor. San Agustín y el Macizo Colombiano, lanzado en la Feria del libro. David emprendió una campaña para solicitar al gobierno y al Icanh la repatriación de las 35 piezas arqueológicas, el derecho de petición va respaldado por firmas de los habitantes de San Agustín. 
dan las instrucciones básicas para conducir los caballos y se cuenta con un seguro que todo caballista debe adquirir al contratar este servicio.

Sin embargo, hace falta más inversión en infraestructura; se realizan campañas promocionales de San Agustín como destino turístico pero los condiciones de algunos caminos y veredas se hallan en muy mal estado y en épocas de lluvia se vuelven intransitables. No hay en San Agustín un plan de accidentes, y si lo hay no es funcional, al menos para los ocurridos en el sector rural. No existen sitios ni personal calificado para prestar los primeros auxilios y no hay un plan de evacuación de heridos ya que en ciertos parajes no llegan los automóviles, solo caballos y motos. La movilización de un herido desde la Pelota al pueblo, puede convertirse en una odisea.

Es urgente promover políticas de conservación del patrimonio arquitectónico de San Agustín, la única calle que se conserva es la de la Locería. Hace poco se aprobó la construcción de un supermercado a un costado del parque principal el cual rompe con la armonía y la tranquilidad que se podía disfrutar en este lugar. ¿Por qué no pensar en la peatonalización de una calle donde queden ubicadas la tiendas de artesanías? Una vía donde se pueda caminar con placidez, adoquinada y embellecida, con sitios donde la palabra y la reflexión tengan acogida. Todo esto integrado a campañas de cultura ciudadana que promuevan el cuidado del pueblo y de los parques, la atención al turista, el respeto y conservación de las costumbres de la región. Enamorar a todos los agustinianos de su tierra, de su legado, de su riqueza cultural para que se conviertan en los principales promotores de su tesoro arqueológico y geográfico.

Por último, se requiere asesoría para que los campesinos puedan beneficiarse del turismo y no se quede todo en manos de los hoteleros y las empresas prestadoras de servicios turísticos. El caso de una campesina que vive de la venta de tintos, aguas aromáticas, jugos, hojaldres y alimentos preparados en un fogón de leña; la 'tiendita' está ubicada estratégicamente, al término del camino que baja del cerro de La Pelota. La propietaria es morena, fuerte, se mueve con rapidez y se esmera por atender bien a sus clientes. Es amable y sencilla, llena de necesidades. El local está construido con los materiales que le brinda el medio: sillas de guadua, tablas y troncos; una mesa adornada con un mantel plástico, fogón de leña, techo de cinc y caucho, pisos de tierra y al fondo el ranchito sin terminar.

En contraste al llegar a la Chaquira encontramos un sitio de descanso, con hermosos quioscos elaborados con guadua, teja, ladrillo; una tienda con neveras, gaseosas, cervezas y paquetes. Ubicado en la planicie antes de emprender el descenso a uno de los lugares más poderosos de todo San Agustín. El lugar pertenece a un suizo que desde hace unos años compró las tierras aledañas a la Chaquira. La atención en el lugar es cortés, pero carece del calor humano, de la sencillez de la gente de la región. Este caso ilustra una de las muchas contradicciones que se presentan en San Agustín.

\section{¿A dónde retornan los ídolos?}

La exposición «El retorno de los ídolos», es una de las actividades centrales de la celebración del Centenario, la cual se realizará en el Museo Nacional de Bogotá desde el 28 de noviembre hasta el 28 de febrero, en ella se exhibirán 20 esculturas, algunas están ubicadas en el recorrido del Bosque de las Estatuas. La actividad está a cargo del Icanh y tiene como objetivo principal promover la cultura del Alto Magdalena y posicionar a San Agustín como destino turístico.

Es de destacar el interés que suscitó la exposición en la región, es la primera vez que el tema Agustiniano es tratado de forma tan copiosa en los periódicos regionales y nacionales ${ }^{5}$. El traslado de las estatuas generó una pasión de

5. Este despliegue periodístico no se dio cuando en el 2011 las esculturas que se encuentran en el Purutal y La Pelota fueron retocadas con vinilo, el Icanh envió expertos para que realizaran el trabajo de limpieza de las tres esculturas, pero el daño ya estaba hecho. Hubo revuelo, pero no el que un acto de esta naturaleza ameritaba, no hubo marchas en defensa de nuestro patrimonio, ni se lanzaron campañas educativas que generaran sentido de pertenencia y de apropiación de las esculturas. ¿Qué pasó? Nada. Silencio y olvido. 
defensa de lo nuestro inusitada: periodistas, profesores y escritores se manifestaron en pro y en contra de esta exposición; el director del IC la defendió con vehemencia y no poca soberbia. Luego de muchas conversaciones y de la socialización de este proyecto con la comunidad que en varias ocasiones ha protagonizado actos de resistencia en defensa de las estatuas; el Concejo de San Agustín, la Asamblea departamental y el Gobernador decidieron apoyar la exposición, a pesar de la inconformidad manifestada por líderes culturales, periodistas y habitantes del municipio.

La exposición será una realidad, las esculturas viajarán de acuerdo al protocolo estipulado, Héctor Llanos, arqueólogo estudioso de la Cultura Agustiniana, será el curador y fue quien realizó la selección de las esculturas; él es la mayor prenda de garantía para que la esculturas regresen sanas y salvas.

Las celebraciones contribuyen a poner en boca de todos, un lugar, un personaje, una obra que forma parte de los bienes culturales de un pueblo, pero como toda celebración -ésta no es la excepción-, en unos meses las esculturas retornarán a su bosque, Preuss será solo un recuerdo que oscilará entre el científico y el traficante, el pueblo seguirá recibiendo turistas en su lucha por sobrevivir en medio del olvido de un país.

Finalmente, no puedo dejar de imaginar la soledad de las esculturas en la fría Bogotá, su timidez violentada por miles de ojos que las interrogarán durante muchos días. Lo que ellos verán no es lo que ellas son. Fuera de su contexto se rompe la unión entre piedra-paisaje que es lo que las fundamenta ¿Cómo sentirlas sin los sonidos, sin el Sol, sin el viento, sin las montañas en las que fueron labradas? Definitivamente, los ídolos no retornan porque nunca se han ido, su espíritu permanece férreo en las montañas, como la piedra en las que fueron creadas.

\author{
Yineth Angulo Cuéllar \\ Profesora catedrática Facultad de Educación. \\ Titular de las Cátedras Agustiniana, Riveriana y Huilense. \\ yineta@hotmail.com
}

\title{
Referencias bibliográficas
}

Caldas, Francisco José. Semanario de la Nueva Granada. Núm. 5. En: http://www.banrepcultural.org/blaavirtual/ historia/semanario/indice.htm, 1808. 1863

Codazzi, Agustín. Antiguedades indijenas. En: http://www.banrepcultural.org/blaavirtual/historia/jeografia/indice.htm,

Friede, Juan. Introducción a la película San Agustín 1942. En: http://www.banrepcultural.org/sites/ default/files/ San_Agustin_200_anos_1790_-_1990._Parte_2.pdf, 1942.

Lasso, Luis Ernesto. Cultura agustiniana: núcleo de resistencia identitaria. Revista Región y Cultura. Núm. 21. Neiva: Oti impresos, 2005.

Llanos, Héctor. Los chamanes jaguares de San Agustín. Génesis de un pensamiento mitopoético. Bogotá, Colombia, 1995.

Neurath, Johannes y JÁUREGUI, Jesús. La expedición de Konrad Theodor Preuss al Nayarit y su contribución a la mexicanística. En http://books.openedition.org/cemcal, 1905-1907.

Pérez de Barradas, José. Arqueología Agustiniana. Bogotá: Imprenta Nacional, 1943.

Preuss, Konrad Theodoro. Arte monumental prehistórico. Bogotá, Colombia: Dirección de divulgación cultural de la Universidad Nacional, 1974. 